\title{
ON THE ZEROS OF CERTAIN CONFLUENT HYPERGEOMETRIC FUNCTIONS
}

\author{
P. WYNN
}

Abstract. The theory of continued fractions is used to derive the following results which hold for $-\frac{1}{2}<\alpha<\infty$ : (1) If

$$
{ }_{1} F_{1}(\alpha ; 2 \alpha+1 ; z)=0 \quad\left[{ }_{1} F_{1}(\alpha+1 ; 2 \alpha+1 ; z)=0\right]
$$

then $\operatorname{Re}(z)>0[<0]$. (It is deduced from this result that if $I_{\alpha-1 / 2}(z)+$ $I_{\alpha+1 / 2}(z)=0$ then $\operatorname{Re}(z)>0$, and it is shown that if $\alpha$ is an integer, an unbounded number of roots of this equation exists.) (2) The roots of the equations

and

$$
{ }_{1} F_{1}(\alpha ; 2 \alpha ; z)={ }_{1} F_{1}(\alpha ; 2 \alpha+1 ; z)
$$

$$
{ }_{1} F_{1}(\alpha ; 2 \alpha ; z)={ }_{1} F_{1}(\alpha+1 ; 2 \alpha+1 ; z)
$$

are identical, pure imaginary, symmetrically distributed about the origin and unbounded in number. (3) Let $C_{n}(z)(n=0,1, \cdots)$ be the successive convergents of the continued fraction associated with $g(z)={ }_{1} F_{1}(\alpha ; 2 \alpha ; z) /{ }_{1} F_{1}(\alpha ; 2 \alpha+1 ; z)$. The roots $z=i y_{v}^{(n)} \quad(\nu=1$, $2, \cdots)$ of the equation $g(z)=C_{n}(z)$ have the same properties as those described in (2). Furthermore, they interlace: subject to a suitable ordering, $y_{v}^{(n)}<y_{v}^{(n+1)}<y_{v+1}^{(n)}(v=1,2, \cdots)$. (A special case of this result concerns the function $e^{z}$ and the convergents of its continued fraction expansion, and is an extension of the formula $e^{2 i v \pi}=1$ $(v=1,2, \cdots)$.)

A number of results concerning the location of the zeros of certain special confluent hypergeometric functions have been obtained by investigating the behaviour of integral expressions involving the functions in question and, in the case of real argument and parameters, by the use of Sturm sequences (for a survey of known theory and associated references to the literature see $[1, \S 6.16])$. In this paper we introduce a new method, based upon the use of continued fractions, to establish that the zeros of certain confluent hypergeometric functions lie in a fixed half-plane and that those of certain combinations of these functions lie on the imaginary axis. (The reader is referred for the analytic theory of continued fractions to the standard works [2] and [3].)

Received by the editors July 7, 1972 and, in revised form, October 26, 1972.

AMS (MOS) subject classifications (1970). Primary 33A30; Secondary 30A08, $30 \mathrm{~A} 22$.

(c) American Mathematical Society 1973 
The results of which we make use are as follows:

Theorem A (Stieltjes [4, Notes 3 and 4]). The function $S(z)$ generates a nonterminating continued fraction of the form

in which

$$
\frac{u_{1}}{1+} \frac{u_{2} z}{1+} \cdots \frac{u_{v} z}{1+} \cdots
$$

$$
0<u_{v}<\infty \quad(v=1,2, \cdots), \quad \lim _{v=\infty} u_{v}=0
$$

if and only if $S(z)=\sum_{v=0}^{\infty} M_{v} /\left(1+z t_{v}\right)$ where $t_{0}=0, M_{0} \geqq 0,0<t_{v}<T<\infty$, $M_{v}>0(v=1,2, \cdots), \sum_{v=0}^{\infty} M_{v}=u_{1}$, the $\left\{t_{v}\right\}$ being distinct; $S(z)$ is then regular at the origin, is meromorphic in any finite region of the z-plane, and has simple poles at the points $z=-t_{v}^{-1}(v=1,2, \cdots)$ on the negative real axis. Expansion (1) converges, in particular, in the neighbourhood of the origin to $S(z)$.

Theorem B (GAuss [5]; see also [3, §89] and [2, §91]). (i) When $\gamma+\nu \neq 0$ $(\nu=0,1, \cdots)$ the function

is an entire function.

$$
{ }_{1} F_{1}(\alpha ; \gamma ; z)=\sum_{\nu=0}^{\infty}\left\{\prod_{r=0}^{\nu-1} \frac{\alpha+\tau}{\gamma+\tau}\right\} \frac{z^{v}}{\nu !}
$$

(ii) The function $f(z)={ }_{1} F_{1}(\alpha ; \gamma ; z) /{ }_{1} F_{1}(\alpha ; \gamma+1 ; z)$ generates the corresponding continued fraction

$$
v_{0}+\frac{v_{1} z}{1+} \frac{v_{2} z}{1+} \cdots \frac{v_{v} z}{1+} \cdots
$$

where $v_{0}=1$,

$$
\begin{aligned}
v_{2 v-1} & =\frac{(\alpha+v-1)}{(\gamma+2 v-2)(\gamma+2 v-1)} \quad(v=1,2, \cdots) \\
v_{2 v} & =-\frac{(\gamma-\alpha+v)}{(\gamma+2 v-1)(\gamma+2 v)}
\end{aligned}
$$

which converges uniformly to $f(z)$ in any finite region of the z-plane from which the zeros of the function ${ }_{1} F_{1}(\alpha ; \gamma+1 ; z)$ have been excluded.

Theorem C (PontrJagin $[6, \S 1])$. Let $P_{m, n}(z, w)$ be a polynomial in the two variables $z$ and $w$ of the form

$$
P_{m, n}(z, w)=\sum_{v=0}^{m} \sum_{\tau=0}^{n} c_{v, r} z^{v} w^{\tau} \quad(1 \leqq m, n<\infty)
$$

where each of the four sets of numbers $c_{0, \tau}, c_{m, \tau}(\tau=0,1, \cdots, n), c_{v, 0}, c_{v, n}$ $(\nu=0,1, \cdots, m)$ contains at least one nonzero member. Then if $c_{m, n}=0$ 
$\left[c_{0, n}=0\right]$ the equation $P_{m, n}\left(z, e^{z}\right)=0$ has an unbounded number of roots with arbitrarily large positive [negative] real part.

Our first main result is

THEOREM 1. If $-\frac{1}{2}<\alpha<\infty$ and

(i) ${ }_{1} F_{1}(\alpha ; 2 \alpha+1 ; z)=0$ then $\operatorname{Re}(z)>0$;

(ii) ${ }_{1} F_{1}(\alpha+1 ; 2 \alpha+1 ; z)=0$ then $\operatorname{Re}(z)<0$.

Proof. Setting $\gamma=2 \alpha$, it follows from clause (ii) of Theorem B that when $-\frac{1}{2}<\alpha<\infty$ the function

$$
g(z)={ }_{1} F_{1}(\alpha ; 2 \alpha ; z) /{ }_{1} F_{1}(\alpha ; 2 \alpha+1 ; z)
$$

generates the corresponding continued fraction (3) with $v_{0}=1, v_{2 v-1}=$ $-v_{2 v}=1 /\{2(2 \alpha+2 v-1)\} \quad(v=1,2, \cdots)$. The even part of expansion (3) (i.e. (see [3, §4]) that continued fraction whose successive convergents are those of even order of expansion (3)) then becomes

where

$$
1+\frac{a z}{1-a z+1+} \frac{u_{1} z^{2}}{1+} \frac{u_{2} z^{2}}{1+} \cdots \frac{u_{v-1} z^{2}}{1+} \cdots
$$

$$
\begin{aligned}
a & =\frac{1}{2(2 \alpha+1)}, \\
u_{v} & =\frac{1}{4(2 \alpha+2 v-1)(2 \alpha+2 v+1)} \quad(v=1,2, \cdots) .
\end{aligned}
$$

From clause (ii) of Theorem B, expansion (5) converges to $g(z)$ in particular in the neighbourhood of the origin. The coefficients $u_{v}(v=1,2, \cdots)$ of formulae (6) satisfy conditions (2). Hence, from Theorem A, the expansion

$$
\frac{u_{1}}{1+} \frac{u_{2} z^{2}}{1+} \cdots \frac{u_{v} z^{2}}{1+} \cdots
$$

converges in the neighbourhood of the origin to $S(z)=\sum_{v=0}^{\infty} M_{v} /\left(1+z^{2} t_{v}\right)$ where the numbers $M_{v}, t_{v}(v=0,1, \cdots)$ are as described in that theorem. In the neighbourhood of the origin

$$
g(z)=1+a z /\left(1-a z+z^{2} S\left(z^{2}\right)\right)
$$

and, by analytic continuation, this relationship holds throughout any finite region of the $z$-plane.

The functions $a z$ and $1-a z+z^{2} S\left(z^{2}\right)$ are analytic when $\operatorname{Re}(z)<0$, and the only singularities of the function $S\left(z^{2}\right)$ upon the line $\operatorname{Re}(z)=0$ are simple poles. Furthermore, since $\operatorname{Re}\left(z^{-1}\right) \leqq 0, \operatorname{Re}(-a)<0, \operatorname{Re}\left\{z S\left(z^{2}\right)\right\} \leqq 0$ when 
$\operatorname{Re}(z) \leqq 0(z \neq 0)$, it follows that the function $z^{-1}-a+z S\left(z^{2}\right)$ is never zero when $\operatorname{Re}(z) \leqq 0$. Hence the function $g(z)$ of formula (4) is analytic when $\operatorname{Re}(z) \leqq 0$.

Now assume that ${ }_{1} F_{1}(\alpha ; 2 \alpha+1 ; z)=0$ for some finite value of $z$ such that $\operatorname{Re}(z) \leqq 0$. Since $g(z)$ is analytic for $\operatorname{Re}(z) \leqq 0$, it follows that ${ }_{1} F_{1}(\alpha ; 2 \alpha ; z)=0$ also. But $[1, \S 6.4]$

$$
2_{1} F_{1}(\alpha ; 2 \alpha ; z)-{ }_{1} F_{1}(\alpha ; 2 \alpha+1 ; z)={ }_{1} F_{1}(\alpha+1 ; 2 \alpha+1 ; z) .
$$

Hence, for this value of $z,{ }_{1} F_{1}(\alpha+1 ; 2 \alpha+1 ; z)=0$. Since $[1, \S 6.4]$

$$
\mathscr{D}_{1} F_{1}(\alpha ; 2 \alpha ; z)=\frac{1}{21} F_{1}(\alpha+1 ; 2 \alpha+1 ; z) \quad\left(-\frac{1}{2}<\alpha<\infty\right)
$$

(where $\mathscr{D}$, now and subsequently, denotes $d / d z$ ) it follows that we have shown that the function ${ }_{1} F_{1}(\alpha ; 2 \alpha ; z)$ and its first derivative vanish for the value of $z$ in question. Using the homogeneous second order differential equation satisfied by the function ${ }_{1} F_{1}(\alpha ; 2 \alpha ; z)[1, \S 6.3]$ it can then be shown that all derivatives of this function vanish at the point in question, and hence that ${ }_{1} F_{1}(\alpha ; 2 \alpha ; z)=0$ identically. This is manifestly untrue, the initial assumption is false and, indeed, if ${ }_{1} F_{1}(\alpha ; 2 \alpha+1 ; z)=0$ then $\operatorname{Re}(z)>0$.

The second result of the theorem is an immediate consequence of the formula [7], [1, §6.3]

$$
{ }_{1} F_{1}(a ; c ; z)=e^{z}{ }_{1} F_{1}(c-a ; c ;-z) \quad(c \neq 0,-1, \cdots) .
$$

Theorem 1 says nothing concerning the existence of the points referred to. Indeed, it is clear that when $\alpha=0$ such points do not exist. We therefore describe two special cases in which the existence of such points can be established and in which something further can be said concerning their distribution.

THEOREM 2. Let $n \geqq 1$ be a fixed finite integer. There exists an unbounded number of values of $z$ having arbitrarily large

(i) positive real part for which ${ }_{1} F_{1}(n ; 2 n+1 ; z)=0$,

(ii) negative real part for which ${ }_{1} F_{1}(n+1 ; 2 n+1 ; z)=0$.

Proof. Set

$$
\psi_{n}(z)=z^{-n-1}\left\{e^{z}-\sum_{v=0}^{n} \frac{z_{v}}{v !}\right\} .
$$

As is easily verified, we have in succession

$$
\begin{aligned}
{ }_{1} F_{1}(n ; 2 n+1 ; z) & =((2 n) ! /(n-1) !) \mathscr{D}^{n-1} \psi_{n}(z), \\
\mathscr{D}^{n-1} \psi_{n}(z) & =(-1)^{n-1} z^{-2 n}\left\{p_{n}(z) e^{z}-q_{n}(z)\right\}
\end{aligned}
$$


where

$$
\begin{aligned}
& p_{n}(z)=\sum_{v=0}^{n-1} \frac{(2 n-v-1) !}{v !(n-v-1) ! n}(-z)^{v}, \\
& q_{n}(z)=\sum_{v=0}^{n} \frac{(2 n-v-1) !}{v !(n-v) !} z^{v} .
\end{aligned}
$$

Since a term involving $z^{n}$ occurs in the expression contained in braces in formula (12), but a term in $e^{z} z^{n}$ is missing, it follows from Theorem $\mathrm{C}$ that this expression has an unbounded number of zeros with arbitrarily large positive real part. That the zeros of the function ${ }_{1} F_{1}(n ; 2 n+1 ; z)$ are confined to the half-plane $\operatorname{Re}(z)>0$ is a consequence of Theorem 1 .

The second result of the theorem is proved in a similar way, noting that ${ }_{1} F_{1}(n+1 ; 2 n+1 ; z)=((2 n) ! / n !) \mathscr{D}^{n} \psi_{n-1}(z)$. We note in passing that in the notation of formula (11)

$\left.\begin{array}{l}\mathscr{D}^{n-1} \psi_{n}(z)=z^{1-n}\left[\Delta_{0}^{n-1}\left\{(n+v) ! \psi_{v+1}(z)\right\}\right] / n ! \\ \mathscr{D}^{n} \psi_{n-1}(z)=z^{-n}\left[\Delta_{0}^{n}\left\{(n+v-1) ! \psi_{v-1}(z)\right\}\right] /(n-1) !\end{array}\right\} \quad(n=1,2, \cdots)$, where the difference operator $\Delta$ is defined by $\Delta^{0} f(v)=f(v)(\nu=0,1, \cdots)$,

and

$$
\Delta^{r+1} f(\nu)=\Delta^{r} f(\nu)-\Delta^{\tau} f(\nu+1) \quad(\nu, \tau=0,1, \cdots)
$$

$$
\Delta_{0}^{r} f(v)=\Delta^{r} f(0) \quad(r=0,1, \cdots) .
$$

The results of Theorems 1 and 2 may be presented in terms of Bessel functions:

THEOREM 3. If, when $-\frac{1}{2}<\alpha<\infty, I_{\alpha-1 / 2}(z)+I_{\alpha+1 / 2}(z)=0$ then (apart from the case $z=0$ when $\left.\alpha>\frac{1}{2}\right) \operatorname{Re}(z)<0$; furthermore, when $\alpha \geqq 1$ is a finite positive integer, this equation has an unbounded number of roots with arbitrarily large negative real part.

Proof. We have $[1, \S 6.9 .1]$

$$
{ }_{1} F_{1}(\alpha ; 2 \alpha ; z)=\left(\frac{1}{2}\right)^{1 / 2-\alpha} \Gamma\left(\alpha+\frac{1}{2}\right) e^{z / 2}\left\{\left(\frac{1}{2} z\right)^{1 / 2-\alpha} I_{\alpha-1 / 2}\left(\frac{1}{2} z\right)\right\},
$$

and $[8, \S 3.71]$

$$
\mathscr{D}\left\{z^{1 / 2-\alpha} I_{x-1 / 2}(z)\right\}=z^{1 / 2-\alpha} I_{\alpha+1 / 2}(z) .
$$

With the help of these two equations and formula (9), we derive

$$
\begin{aligned}
& I_{\alpha+1 / 2}(z)+I_{\alpha-1 / 2}(z) \\
& \quad=\left(\left(\frac{1}{2} z\right)^{\alpha-1 / 2} e^{-z} / \Gamma\left(\alpha+\frac{1}{2}\right)\right)_{1} F_{1}(\alpha+1 ; 2 \alpha+1 ; 2 z) \quad\left(-\frac{1}{2}<\alpha<\infty\right) .
\end{aligned}
$$

The first result of the theorem follows immediately from clause (ii) of Theorem 1. 
The second result may be deduced from Theorem 2 . It may also, with the help of Theorem $\mathrm{C}$, be deduced from first principles using the fact [8, §3.71] that when $\alpha \geqq 1$ is a finite integer

where

$$
I_{\alpha-1 / 2}(z)=\left\{e^{2 z} \sum_{r=0}^{\alpha-1} \phi_{\alpha, r}(2 z)^{r}-\sum_{r=0}^{\alpha-1}(-1)^{r} \phi_{\alpha, r}(2 z)^{r}\right\} /\left\{e^{z}(2 z)^{\alpha-1} \sqrt{ }(2 \pi z)\right\}
$$

$$
\begin{array}{r}
\phi_{\alpha, r}=\left[\prod_{v=1}^{\alpha-r-1}\left\{(2 v-1)^{2}-(2 \alpha-1)^{2}\right\}\right] /\left\{(\alpha-r-1) ! 4^{\alpha-r-1}\right\} \\
(r=0,1, \cdots, \alpha-1) .
\end{array}
$$

Our second main result is

THEOREM 4. Let $-\frac{1}{2}<\alpha<\infty$. The roots of the equations

$$
\begin{aligned}
& { }_{1} F_{1}(\alpha ; 2 \alpha ; z)={ }_{1} F_{1}(\alpha ; 2 \alpha+1 ; z), \\
& { }_{1} F_{1}(\alpha ; 2 \alpha ; z)={ }_{1} F_{1}(\alpha+1 ; 2 \alpha+1 ; z),
\end{aligned}
$$

are identical, pure imaginary, symmetrically distributed about the origin, and unbounded in number.

Proof. From formulae (4) and (7), we have

$$
\begin{aligned}
z^{2} S\left(z^{2}\right) & =\frac{1-g(z)+a z g(z)}{g(z)-1} \\
& =\frac{{ }_{1} F_{1}(\alpha ; 2 \alpha+1 ; z)+(a z-1)_{1} F_{1}(\alpha ; 2 \alpha ; z)}{{ }_{1} F_{1}(\alpha ; 2 \alpha ; z)-{ }_{1} F_{1}(\alpha ; 2 \alpha+1 ; z)}
\end{aligned}
$$

where $a=1 /\{2(2 \alpha+1)\}$. Setting

$$
h(z)=z^{2 \alpha}\left\{{ }_{1} F_{1}(\alpha ; 2 \alpha ; z)-{ }_{1} F_{1}(\alpha ; 2 \alpha+1 ; z)\right\}
$$

and using [1, §6.4], it is easily verified that $\mathscr{D} h(z)=\frac{1}{2} z^{2 \alpha}{ }_{1} F_{1}(\alpha+1 ; 2 \alpha+1 ; z)$. Hence, from equation (8),

$$
\begin{aligned}
z^{2 \alpha}{ }_{1} F_{1}(\alpha ; 2 \alpha ; z) & =2 \mathscr{D} h(z)-h(z), \\
\frac{1}{2} z^{2 \alpha}{ }_{1} F_{1}(\alpha ; 2 \alpha+1 ; z) & =\mathscr{D} h(z)-h(z),
\end{aligned}
$$

and formula (18) may be written as $z^{2} S\left(z^{2}\right)=(2 a z \mathscr{D} h(z)-(1+a z) h(z)) / h(z)$. The expressions in the numerator and denominator of the fraction upon the right-hand side of this equation represent entire functions. These functions do not vanish for the same value of $z$, for if they were to do so, the function ${ }_{1} F_{1}(\alpha ; 2 \alpha ; z)$ and its first derivative $\frac{1}{21} F_{1}(\alpha+1 ; 2 \alpha+1 ; z)$ would also vanish simultaneously, implying that the former is identically zero. It was shown in the proof of Theorem 1 that the function $S\left(z^{2}\right)$ is analytic for $\operatorname{Re}(z) \neq 0$, and that its poles are unbounded in number, pure 
imaginary and symmetrically distributed about the origin. The poles of $S\left(z^{2}\right)$ occur at the zeros of $h(z)$. We have disposed of what has to be proved concerning equation (16).

That equations (16) and (17) have identical roots follows immediately from equation (10).

The results of Theorem 4 may, with the help of relationships (14) and (15), also be presented in terms of Bessel functions. However, the sole product of this change of notation is that, if $0<\beta<\infty$, the zeros of $J_{\beta}(z)$ are real, symmetrically distributed about the origin, and unbounded in number, a result which is in any case well known to hold for $-1 \leqq \beta<\infty$ [9], [8, §15.27].

THEOREM 5. Let the function $g(z)$ and the numbers $a$ and $u_{v}(v=1,2, \cdots)$ be as defined in Theorem 1. Set

$$
\begin{aligned}
& C_{0}(z)=1, \quad C_{1}(z)=1+a z /(1-a z), \\
& C_{n}(z)=1+\frac{a z}{1-a z+1+} \frac{u_{1} z^{2}}{1+} \cdots \frac{u_{n-1} z^{2}}{1} \quad(n=2,3, \cdots) .
\end{aligned}
$$

Then for a finite integer $n \geqq 0$, the roots $z=i y_{v}^{(n)}(\nu=0,1, \cdots)$ of the equation

$$
g(z)=C_{n}(z)
$$

are pure imaginary, symmetrically distributed about the origin and unbounded in number. One of these roots is at the origin, the rest are bounded away from it. Furthermore, these roots interlace: if the roots on the nonnegative imaginary axis are ordered according to the scheme $y_{0}^{(n)}=0, y_{1}^{(n)}<y_{2}^{(n)}<\cdots$, we have $y_{v}^{(n)}<y_{v}^{(n+1)}<y_{v+1}^{(n)}(v=1,2, \cdots)$.

Proof. We first remark that $g(0)=C_{n}(0)=1$ : equation (20) has the root $z=0$.

From Theorem A, the continued fraction

$$
\frac{u_{r+1}}{1+} \frac{u_{r+2} z}{1+} \ldots \frac{u_{r+v} z}{1+} \ldots
$$

converges in the neighbourhood of the origin to the function

$$
S^{(r)}(z)=\sum_{v=0}^{\infty} \frac{M_{v}^{(r)}}{1+z t_{v}^{(r)}}
$$

where $t_{0}^{(r)}=0, M_{0}^{(r)} \geqq 0,0<\cdots<t_{2}^{(r)}<t_{1}^{(r)}<T^{(r)}<\infty, M_{v}^{(r)}>0(v=1,2, \cdots)$, $\sum_{v=0}^{\infty} M_{v}^{(r)}=u_{r+1}<\infty$. The function $S^{(r)}(z)$ has simple poles at the points $z=x_{v}^{(r)}=-1 / t_{v}^{(r)}(v=1,2, \cdots)$ where, setting $X^{(r)}=-1 / T^{(r)}, \cdots<x_{2}^{(r)}<$ $x_{1}^{(r)}<X^{(r)}<0$. For real values of $z \neq x_{v}^{(r)}(\nu=1,2, \cdots)$ we have

$$
\mathscr{D} S^{(r)}(z)=-\sum_{v=0}^{\infty} \frac{M^{(r)} t_{v}^{(r)}}{\left(1+z t_{v}^{(r)}\right)^{2}}<0 .
$$


For real values of $z$, the function $S^{(r)}(z)$ is real valued, and is positive for nonnegative values of $z$; between its successive poles its gradient is negative. It follows that exactly one zero $z=\hat{x}_{v}^{(r)}$ of $S^{(r)}(z)$ lies between each pair of neighbouring poles of this function: subject to a suitable ordering, $x_{v+1}^{(r)}<\hat{x}_{v}^{(r)}<x_{v}^{(r)}(v=1,2, \cdots)$. The functions $\left\{S^{(r)}(z)\right\}$ satisfy the equation $S^{(r)}(z)=u_{r+1} /\left(1+z S^{(r+1)}(z)\right)$. The poles $\left\{x_{v}^{(r+1)}\right\}$ of $S^{(r+1)}(z)$ correspond to the zeros of $S^{(r)}(z)$ : we have $x_{v+1}^{(r)}<x_{v}^{(r+1)}<x_{v}^{(r)}(v=1,2, \cdots)$.

In conjunction with formulae (19), we have

$$
g(z)=\frac{a z}{1-a z+1+} \frac{u_{1} z^{2}}{1+} \cdots \frac{u_{n-1} z^{2}}{1+} \frac{u_{n} z^{2}}{1+z^{2} S^{(n)}\left(z^{2}\right)} .
$$

When $S^{(n)}\left(z^{2}\right)=\infty$, equation (20) is satisfied. This occurs when $z^{2}=x_{v}^{(n)}$ $(v=1,2, \cdots)$, i.e. when $z= \pm i y_{v}^{(n)}(v=1,2, \cdots)$ where $y_{v}^{(n)^{2}}=-x_{v}^{(n)}$ $(\nu=1,2, \cdots)$. The stated properties of the numbers $\left\{y_{v}^{(n)}\right\}$ follow from those already derived for the numbers $\left\{x_{v}^{(n)}\right\}$.

It is clear that the argument used in the above proof can be applied to derive a similar result concerning any function which generates a continued fraction having a tail which is of the form described in Theorem A, or which has a similar form.

Corollary. Let $C_{n}(z)(0 \leqq n<\infty)$ be the nth convergent of the continued fraction expansion [10], [2, §48], [3, §91]

$$
1+\frac{z}{1-\frac{1}{2} z+} \frac{z^{2} / 12}{1+} \frac{z^{2} / 60}{1+} \ldots \frac{z^{2} /\left\{4\left(4 v^{2}-1\right)\right\}}{1+} \cdots
$$

of $e^{\grave{z}}$ (or, equivalently: let $C_{n}(z)$ be the Padé quotient $R_{n, n}(z)$ derived from the power series expansion of $\left.e^{z}\right)$; then the roots of the equation

$$
e^{z}=C_{n}(z)
$$

possess all the properties of those relating to equation (20) described in Theorem 5.

Proof. As $\alpha$ tends to zero, the function $g(z)$ of formula (4) tends to $\frac{1}{2}\left(e^{z}+1\right)$, and the continued fraction (5) tends to the form (21) in which the partial numerator $z$ is replaced by $\frac{1}{2} z$. We denote the successive convergents of this expansion by $\hat{C}_{n}(z)(n=0,1, \cdots)$. Theorem 5 then concerns the equation $\frac{1}{2}\left(e^{z}+1\right)=\hat{C}_{n}(z)$. This equation may be rearranged in the form (22).

The above corollary may be regarded as being an extension of the result $e^{2 i v \pi}=1(\nu= \pm 0,1, \cdots)$, and Theorem 5 as being an extension of this corollary.

The successive convergents of expansion (21) may be expressed in closed 
form [11], [12]: in the notation of formulae (13), we have

$$
C_{n}(z)=\left(p_{n}(-z)+q_{n}(z)\right) /\left(p_{n}(z)+q_{n}(-z)\right) \quad(n=0,1, \cdots) .
$$

Equation (22), expressed in the form $e^{i y}=C_{n}(i y)$, involves complex numbers. However, by rewriting it in a form involving $\left(e^{i y}-1\right) /\left(e^{i v}+1\right)$ and using formulae (13) and (23), we may derive an equation involving real numbers only; it is

$$
\tan \left(\frac{1}{2} y\right)=\left(\chi_{n}(y)+\omega_{n}(y)\right) /\left(\eta_{n}(y)+\sigma_{n}(y)\right) \quad(n=0,1, \cdots)
$$

where

$$
\begin{aligned}
& \chi_{n}(y)=\sum_{v=0}^{[(n-2) / 2]} \frac{(2 n-2 v-2) !(-1)^{v}}{(2 v+1) !(n-2 v-2) ! n} y^{2 v+1}, \\
& \omega_{n}(y)=\sum_{v=0}^{[(n-1) / 2]} \frac{(2 n-2 v-2) !(-1)^{v}}{(2 v+1) !(n-2 v-1) !} y^{2 v+1}, \\
& \eta_{n}(y)=\sum_{v=0}^{[(n-1) / 2]} \frac{(2 n-2 v-1) !(-1)^{v}}{(2 v) !(n-2 v-1) ! n} y^{2 v}, \\
& \sigma_{n}(y)=\sum_{v=0}^{[n / 2]} \frac{(2 n-2 v-1) !(-1)^{v}}{(2 v) !(n-2 v) !} y^{2 v} .
\end{aligned}
$$

Denoting the expression upon the right-hand side of equation (23) by $\xi_{n}(y)$ we have, for $n$ fixed and large values of $y, \xi_{n}(y) \sim-n(n+1) / y$ when $n$ is even, and $\xi_{n}(y) \sim y /\{n(n+1)\}$ when $n$ is odd. Denoting the root of equation (24) in the interval $((2 r-1) \pi,(2 r+1) \pi)$ by $\hat{y}_{n, r}$ we have, for large values of $r, \hat{y}_{n, r} \sim 2 r \pi$ when $n$ is even and $\hat{y}_{n, r} \sim(2 r+1) \pi$ when $n$ is odd.

\section{REFERENCES}

1. A. Erdelyi et al., Higher transcendental functions. The hypergeometric function, Legendre functions, vol. 1, McGraw-Hill, New York, 1953. MR 15, 419.

2. O. Perron, Die Lehre von den Kettenbrüchen. Dritte, Verbesserte und erweiterte Aufl. Band II. Analytisch-funktionentheoretische Kettenbruche. Teubner Verlagsgesellschaft, Stuttgart, 1957. MR 19, 25.

3. H. S. Wall, Analytic theory of continued fractions, Van Nostrand, Princeton, N.J., 1948. MR 10, 32.

4. T. J. Stieltjes, Recherches sur les fractions continues, Ann. Fac. Sci. Toulouse 8 (1894), 1-122; 9 (1895), 1-47 (also: Oeuvres complètes de Thomas Jan Stieltjes, vol. 2, Nordhoff, Groningen, 1918, pp. 402-566).

5. C. F. Gauss, Disquisitiones generales circa seriem infinitan, Commentationes societatis scientiarum Goettingensis recentiores 2 (1813),1-46; Werke, 3 (1876),123-162.

6. L. Pontrjagin, On the zeros of certain transcendental functions, Izv. Akad. Nauk SSSR Ser. Mat. 6 (1942), 115-134 (Russian); English transl., Amer. Math. Soc. Transl. (2) 1 (1955), 95-110. MR 4, 214; MR 17, 471. 
7. E. E. Kummer, De integralibus quibisdam definitis et seriebus infinitis, J. Reine Angew. Math. 17 (1837), 228-242.

8. G. N. Watson, Theory of Bessel functions, 2nd ed., Cambridge Univ. Press, Cambridge; Macmillan, New York, 1944. MR 6, 64.

9. A. Hurwitz, Ueber die Nulstellen der Bessel'sche Funktion, Math. Ann. 33 (1889), 246-266.

10. J. L. Lagrange, Sur l'usage des fractions continues dans le calcul intégral, Nouveaux mémoires de l'académie royale des sciences et belles-lettres de Berlin 7 (1776), 236-264; Oeuvres 4 (1869), 301-332.

11. G. Darboux, Sur les développements en série des fonctions d'une seule variable, J. Math. Pures Appl., 2 (1876), 291-312.

12. H. Padé, Mémoire sur les développements en fractions continues de la fonction exponientielle pouvant servir d'introduction à la théorie des fractions continues algébriques, Ann. Sci. École Norm. Sup. 16 (1899), 395-426.

Centre de Recherches Mathématiques, Université de Montréal, Montréal, QuÉBEC, CANADA 